\title{
Fixed Point Theorem for Meir-Keeler Type Function in $b_{2}$-Metric Spaces
}

\author{
Zhuoyi Tian, Jinxing Cui, Linan Zhong* \\ Department of Mathematics, Yanbian University, Yanji, China \\ Email: *zhonglinan2000@126.com
}

How to cite this paper: Tian, Z.Y., Cui, J.X. and Zhong, L.N. (2019) Fixed Point Theorem for Meir-Keeler Type Function in $b_{2}$-Metric Spaces. Open Access Library Journal, 6: e5973.

https://doi.org/10.4236/oalib.1105973

Received: December 3, 2019

Accepted: December 21, 2019

Published: December 24, 2019

Copyright (๑) 2019 by author(s) and Open Access Library Inc.

This work is licensed under the Creative Commons Attribution International License (CC BY 4.0).

http://creativecommons.org/licenses/by/4.0/

\begin{abstract}
In this paper, we prove fixed point theorems of a generalization which is related to the concept of Meir-Keeler function in a complete $b_{2}$-metric space. And we know it extends and generalizes some known results in metric space to $b_{2}$-metric space.
\end{abstract}

\section{Subject Areas}

Function Theory, Functional Analysis, Mathematical Analysis

\section{Keywords}

Fixed Point, $b_{2}$-Metric Space, Meir-Keeler Function

\section{Introduction}

Many mathematicians have studied fixed point theory over the last several decades since Banach contraction principle [1] was introduced in 1992. The notion of Meir-Keeler function [2] was introduced in 1969. Then the concept of weaker Meir-Keeler function [3] was introduced by Chi-Ming Chen in 2012. And in this paper, we establish fixed point for Meir-Keeler function and weaker Meir-Keeler function in a complete new type of generalized matric space, which is called by $b_{2}$-metric space, and this space was generalized from both 2-metric space [4] [5] [6] and b-metric space [7] [8].

\section{Preliminaries}

Throughout this paper $N$ will denote the set of all positive integers and $R$ will denote the set of all real numbers.

Before stating our main results, some necessary definitions might be introduced as follows. 
Definition 2.1 [2] Let $X$ be a nonempty subsets, $m \in N$ and $f: X \rightarrow X$ an operator. Then $X=\bigcap_{i=1}^{m} A_{i}$ is called a cyclic representation of $X$ with respect to $f$ if

1) $A_{i}, i=1,2, \cdots, m$ are empty subsets of $X$,

2) $f\left(A_{i}\right) \subset A_{2}, f\left(A_{2}\right) \subset A_{3}, \cdots, f\left(A_{m-1}\right) \subset A_{m}, f\left(A_{m}\right) \subset A_{1}$.

Definition 2.2 [2] A function $\phi:(0 \rightarrow \infty] \rightarrow(0 \rightarrow \infty$ ] is said to be a Meir-Keeler function if for each $\eta>0$, there exists $\delta>0$ such that for each $t \in(0 \rightarrow \infty]$ with $\eta \leq t \leq \eta+\delta$, we have $\phi(t)<\eta$.

Definition 2.3 [3] We call $\phi:(0 \rightarrow \infty] \rightarrow(0 \rightarrow \infty$ ] a weak Meir-Keeler function if for each $\eta>0$ such that for each $t \in(0 \rightarrow \infty]$ with $\eta \leq t \leq \eta+\delta$, there exists $n_{0} \in N$ such that $\phi^{n_{0}}(t)<\eta$.

Definition 2.4 [4] [5] [6] Let $X$ be an nonempty set and let $d: X \times X \times X \rightarrow R$ be a map satisfying the following conditions:

1) For every pair of distinct points $x, y \in X$, there exists a point $z \in X$ such that $d(x, y, z) \neq 0$.

2) If at least two of three points $x, y, z$ are the same, then $d(x, y, z)=0$,

3) The symmetry:

$d(x, y, z)=d(x, z, y)=d(y, x, z)=d(y, z, x)=d(z, x, y)=d(z, x, y)$ for all $x, y, z \in X$.

4)The rectangle inequality:

$d(x, y, z) \leq d(x, y, a)+d(y, z, a)+d(z, x, a)$ for all $x, y, z, a \in X$.

Then $d$ is called a 2 metric on $X$ and $(X, d)$ is called a 2 metric space.

Definition 2.5 [7] [8] Let $X$ be a nonempty set and $s \geq 1$ be a given real number. A

function $d: X \times X \rightarrow R^{+}$is a $b$ metric on $X$ if for all $x, y, z \in X$, the following conditions hold:

1) $d(x, y)=0$ if and only if $x=y$.

2) $d(x, y)=d(y, x)$.

3) $d(x, y) \leq s[d(x, y)+d(y, z)]$.

In this case, the pair $(X, d)$ is called a b metric space.

Definition 2.6 [9] Let $X$ be a nonempty set, $s \geq 1$ be a real number and let $d: X \times X \times X \rightarrow R$ be a map satisfying the following conditions:

1) For every pair of distinct points $x, y \in X$, there exists a point $z \in X$ such that $d(x, y, z) \neq 0$.

2) If at least two of three points $x, y, z$ are the same, then $d(x, y, z)=0$,

3) The symmetry:

$d(x, y, z)=d(x, z, y)=d(y, x, z)=d(y, z, x)=d(z, x, y)=d(z, x, y)$ for all $x, y, z \in X$.

4) The rectangle inequality:

$d(x, y, z) \leq s[d(x, y, a)+d(y, z, a)+d(z, x, a)]$, for all $x, y, z, a \in X$.

Then $d$ is called a $b_{2}$ metric on $X$ and $(X, d)$ is called a $b_{2}$ metric space with parameter $s$. Obviously, for $s=1, b_{2}$ metric reduces to 2-metric.

Definition 2.7 [9] Let $\left\{x_{n}\right\}$ be a sequence in a $b_{2}$ metric space $(X, d)$. 
1) A sequence $\left\{x_{n}\right\}$ is said to be $b_{2}$-convergent to $x \in X$, written as $\lim _{n \rightarrow \infty} x_{n}=x$, if all $a \in X \quad \lim _{n \rightarrow \infty} d\left(x_{n}, x, a\right)=0$.

2) $\left\{x_{n}\right\}$ is Cauchy sequence if and only if $d\left(x_{n}, x_{m}, a\right) \rightarrow 0$, when $n, m \rightarrow \infty$. for all $a \in X$.

3) $(X, d)$ is said to be complete if every $b_{2}$-Cauchy sequence is a $b_{2}$-convergent sequence.

Definition 2.8 [9] Let $(X, d)$ and $\left(X^{\prime}, d^{\prime}\right)$ be two $b_{2}$-metric spaces and let $f: X \rightarrow X^{\prime}$ be a mapping. Then $f$ is said to be $b_{2}$-continuous, at a point $z \in X$ if for a given $\varepsilon>0$, there exists $\delta>0$ such that $x \in X$ and $d(z, x, a)<\delta$ for all $a \in X$ imply that $d^{\prime}(f z, f x, a)<\varepsilon$. The mapping $f$ is $b_{2}$-continuous on $X$ if it is $b_{2}$-continuous at all $z \in X$.

Definition 2.9 [9] Let $(X, d)$ and $\left(X^{\prime}, d^{\prime}\right)$ be two $b_{2}$-metric spaces. Then a mapping $f: X \rightarrow X^{\prime}$ is $b_{2}$-continuous at a point $x \in X^{\prime}$ if and only if it is $b_{2}$-sequentially continuous at $x$, that is, whenever $\left\{x_{n}\right\}$ is $b_{2}$-convergent to $x$, $\left\{f x_{n}\right\}$ is $b_{2}$-convergent to $f(x)$.

\section{Main Results}

In this section, we give and prove a generalization of the Meir-Keeler fixed point theorem [2].

Theorem 3.1. Let $(X, d)$ be a complete $b_{2}$-metric space and let $f$ be a mapping on $X$, for each $\varepsilon>0$, there exists $\delta \in(s \varepsilon,(2 s-1) \varepsilon)$ such that

(a) $\frac{1}{2 s} d(x, f x, a)<d(x, y, a)$ and $d(x, y, a)<\varepsilon+\delta$ imply $d(f x, f y, a) \leq \varepsilon$

(b) $\frac{1}{2 s} d(x, f x, a)<d(x, y, a) \quad$ implies $\quad d(f x, f y, a)<d(x, y, a)$ for all $x, y \in X$. Then there exists a unique fixed point $z$ of $f$. Moreover $\lim _{n \rightarrow \infty} f^{n} x=z$ for all $x \in X$.

Proof If $f x \neq x$, then we can easily get that $d(x, f x, a)<2 s d(x, f x, a)$. So, by hypothesis, $d\left(f x, f^{2} x, a\right)<d(x, f x, a)$ holds for all $x \in X$ with $f x \neq x$. We also get

$$
d\left(f x, f^{2} x, a\right) \leq d(x, f x, a) \text { for all } x \in X
$$

Fix point $x_{0}$ in $X$ and define a sequence $\left\{x_{n}\right\}$ in $X$ by $x_{n+1}=f x_{n}=f^{n} x_{0}$ for $n \in N$. From the above (3.1) we get $d\left(x_{n}, x_{n+1}, a\right) \leq d\left(x_{n-1}, x_{n}, a\right)$, so we know that $\left\{d\left(x_{n}, x_{n+1}, a\right)\right\}$ is a decreasing sequence, and the sequence $\left\{d\left(x_{n}, x_{n+1}, a\right)\right\}$ converges to some $\beta \geq 0$. We assume that $\beta>0$, then we know that $d\left(x_{n}, x_{n+1}, a\right)>\beta$ for every $n \in N$, then there exists $\delta$ such that (a) is true with $\varepsilon=\beta$, for the definition of $\beta$, there exists $i \in N$ such that $d\left(x_{i}, x_{i+1}, a\right)<\beta+\delta$, so we have $d\left(x_{i+1}, x_{i+2}, a\right) \leq \beta$, which is a contraction. Therefore $\beta=0$, and that is:

$$
\lim _{n \rightarrow \infty} d\left(x_{n}, x_{n+1}, a\right)=0 .
$$

Now we show that $d\left(x_{i}, x_{j}, x_{k}\right)=0$.

From part 2 of Definition 2.6, the equation $d\left(x_{m}, x_{m}, x_{m-1}\right)=0$ is obtained. 
Since $\left\{d\left(x_{n}, x_{n+1}, a\right)\right\}$ is decreasing, if $d\left(x_{n-1}, x_{n}, a\right)=0$, then $d\left(x_{n}, x_{n+1}, a\right)=0$, then it is easy to get

$$
d\left(x_{n}, x_{n+1}, x_{m}\right)=0 \text {, for all } n+1 \geq m .
$$

For $0 \leq n+1<m$, we get $m-1 \geq n+1$ and that is $m-2 \geq n$, from (3.2)

$$
d\left(x_{m-1}, x_{m}, x_{n+1}\right)=d\left(x_{m-1}, x_{m}, x_{n}\right)=0,
$$

From (3.2) and triangular inequality,

$$
\begin{aligned}
& d\left(x_{n}, x_{n+1}, x_{m}\right) \\
& \leq s d\left(x_{n}, x_{n+1}, x_{m-1}\right)+s d\left(x_{n+1}, x_{m}, x_{m-1}\right)+d\left(x_{m}, x_{n}, x_{m-1}\right) \\
& =s d\left(x_{n}, x_{n+1}, x_{m-1}\right) .
\end{aligned}
$$

And since $d\left(x_{n}, x_{n+1}, x_{n+1}\right)=0$, and from the inequality above,

$$
d\left(x_{n+1}, x_{n}, x_{m}\right) \leq s^{m-n-1} d\left(x_{n+1}, x_{n+1}, x_{n}\right)=0 \text {, for all } 0 \leq n+1 \leq m .
$$

Now for all $i, j, k \in N$, the condition of $j>i$ is considered here, from the above equation

$$
d\left(x_{j-1}, x_{j}, x_{i}\right)=d\left(x_{k}, x_{j-1}, x_{j}\right)=0
$$

From (3.5) and triangular inequality, therefore

$$
\begin{aligned}
d\left(x_{i}, x_{k}, x_{j}\right) & \leq s\left[d\left(x_{i}, x_{j}, x_{j-1}\right)+d\left(x_{j}, x_{k-1}, x_{k}\right)+d\left(x_{i}, x_{j-1}, x_{k}\right)\right] \\
& \leq \cdots \\
& \leq s^{j-1} d\left(x_{i}, x_{k}, x_{i}\right) \\
& =0
\end{aligned}
$$

In conclusion, the result below is true

$$
d\left(x_{j}, x_{k}, x_{i}\right)=0 \text {, for all } i, j, k \in N .
$$

Now we fix $\varepsilon>\frac{\delta}{2 s-1}$, then there exists $\delta$ such that (a) is true. Let $N_{1} \in N$ such that

$$
d\left(x_{n}, x_{n+1}, a\right)<\frac{\delta-(s-1) \varepsilon}{s}, \text { for all } n \in N \text { with } n \geq N_{1} .
$$

Now we will show that

$$
d\left(x_{k}, x_{k+m}, a\right)<\varepsilon+\delta \text { for } m \in N
$$

By induction, when $m=1$, it is true for (3.8). We assume that (3.8) holds for some $m \in N$.

In one case $d\left(x_{k}, x_{k+m}, a\right) \leq \varepsilon$, we have

$$
d\left(x_{k}, x_{k+m+1}, a\right) \leq s\left[d\left(x_{k}, x_{k+m}, a\right)+d\left(x_{k+m}, x_{k+m+1}, a\right)+d\left(x_{k}, x_{k+m+1}, x_{k+m}\right)\right]
$$

From (3.6) and (3.7) we have

$$
\begin{aligned}
d\left(x_{k}, x_{k+m+1}, a\right) & \leq s\left[d\left(x_{k}, x_{k+m}, a\right)+d\left(x_{k+m}, x_{k+m+1}, a\right)\right] \\
& \leq s \varepsilon+s \frac{\delta-(s-1) \varepsilon}{s} \\
& \leq \varepsilon+\delta
\end{aligned}
$$


In other case, where $\varepsilon<d\left(x_{k}, x_{k+m}, a\right)<\varepsilon+\delta$, since

$$
d\left(x_{k}, x_{k+1}, a\right)<\frac{\delta-(s-1) \varepsilon}{s}<\varepsilon<d\left(x_{k}, x_{k+m}, a\right)<2 s d\left(x_{k}, x_{k+m}, a\right)
$$

We get $d\left(x_{k+1}, x_{k+m+1}, a\right) \leq \varepsilon$ and then we have

$$
d\left(x_{k}, x_{k+m+1}, a\right) \leq s\left[d\left(x_{k}, x_{k+m}, a\right)+d\left(x_{k+m}, x_{k+m+1}, a\right)\right] \leq \varepsilon+\delta
$$

So for (3.9) and (3.10), (3.8) is true for every $m \in N$. Therefore we have $\lim _{n \rightarrow \infty} \sup d\left(x_{n}, x_{m}, a\right)=0$, for all $n<m$. This shows that $\left\{x_{n}\right\}$ is a Cauchy sequence.

Since $X$ is complete, there exists a point $z \in X$ such that sequence $\left\{x_{n}\right\}$ converges to it. From the following two respectively cases, we will show that this point is a fixed point for $f$.

Case one: There exists $u \in N$ such that $x_{u}=x_{u+1}$.

Case two: $x_{n} \neq x_{n+1}$, for all $n \in N$.

In the first case, we know that $x_{n}=x_{u}$ for $n \geq u, u \in N$. Since $\left\{x_{n}\right\} \rightarrow z$ as $n \rightarrow \infty$, then we get $x_{n}=z$ for $n \geq u, n \in N$. This prove that $f z=z$.

In the second case, we know that $x_{n} \neq x_{n+1}=f x_{n}$, for all $n \in N$, so we get sequence $\left\{d\left(x_{n}, x_{n+1}, a\right)\right\}$ is strictly decreasing. If we assume that

$$
d\left(x_{n}, x_{n+1}, a\right) \geq 2 s d\left(x_{n}, z, a\right) \text { and } d\left(x_{n+1}, x_{n+2}, a\right) \geq 2 s d\left(x_{n+1}, z, a\right)
$$

for some $n \in N$. For the first inequality of the above assumption, we choose $a=x_{n+1}$, then we have

$$
d\left(x_{n}, x_{n+1}, z\right)=0
$$

Then we have

$$
\begin{aligned}
d\left(x_{n}, x_{n+1}, a\right) & \leq s\left[d\left(x_{n}, z, a\right)+d\left(x_{n+1}, z, a\right)+d\left(x_{n}, x_{n+1}, a\right)\right] \\
& \leq s\left[d\left(x_{n}, z, a\right)+d\left(x_{n+1}, z, a\right)\right] \\
& \leq \frac{d\left(x_{n}, x_{n+1}, a\right)+d\left(x_{n+1}, x_{n+2}, a\right)}{2} \\
& <d\left(x_{n}, x_{n+1}, a\right) .
\end{aligned}
$$

This is a contraction. So we get either

$d\left(x_{n}, x_{n+1}, a\right)<2 s d\left(x_{n}, z, a\right) \quad$ or $\quad d\left(x_{n+1}, x_{n+2}, a\right)<2 s d\left(x_{n+1}, z, a\right) \quad$ for $\quad$ all $n \in N$. Since $x_{n} \rightarrow z$ as $n \rightarrow \infty$, the above inequality prove that there exists a sub sequence of sequence $\left\{x_{n}\right\}$, which converges to $f z$. This shows that $z$ is a fixed point of $f$. Next we prove that $z$ is the unique fixed point of $f$. Suppose that $z$ and $y$ are two different fixed point of $f$, from the assumption of this theorem, we get

$$
\begin{gathered}
d(z, f z, a)=0<2 d(y, z, a) \text { from the above inequality we have } \\
d(z, y, a)=d(f x, f y, a)<d(y, z, a)
\end{gathered}
$$

This is a contraction. Hence $z$ is a unique fixed point of $f$.

In this section, we prove a fixed point theory for the cyclic weaker Meir-Keeler function in $b_{2}$-metric space. Now we give some comments as follows:

$\Omega=\omega:(0 \rightarrow \infty] \rightarrow(0 \rightarrow \infty]$ is a set, where $\omega$ is a weaker Meir-Keeler func- 
tion and satisfying the following conditions:

$\left(\omega_{1}\right) \omega(t)>0$ for $t>0$, and $\omega(0)=0$;

$\left(\omega_{2}\right)$ For all $t \in(0 \rightarrow \infty],\left\{\omega^{n}(t)\right\}_{n \in N}$ is decreasing;

$\left(\omega_{3}\right)$ For $t_{n} \in(0 \rightarrow \infty]$, if $\lim _{n \rightarrow \infty} t_{n}=\gamma$, then $\lim _{n \rightarrow \infty} \omega\left(t_{n}\right)<\gamma$.

$\Theta=\theta:[0 \rightarrow \infty) \rightarrow[0 \rightarrow \infty)$, where $\theta$ is a non-increasing and continuous function with $\theta(t)>0$ for all $t>0$ and $\theta(0)=0$.

We now introduce the following definition of cyclic weaker $(\omega, \theta)$ -contraction mapping in $b_{2}$-metric space:

Definition 3.2 Let $(X, d)$ be a $b_{2}$-metric space, $A_{1}, A_{2}, \cdots, A_{m}$ are all nonempty subsets of $X=\bigcup_{i=1}^{m} A_{i}$. A mapping $f: X \rightarrow X$ is said to be cyclic weaker $(\omega, \theta)$-contraction in $b_{2}$-metric space if satisfying the following condition:

1) $X=\bigcup_{i=1}^{m} A_{i}$ with respect of $f$, it is a cyclic representation of $X$.

2) $i=1,2, \cdots, m$, for any $x \in A_{i}, y \in A_{i+1}$, such that

$d(f x, f y, a) \leq \omega(d(x, y, a))-\theta(d(x, y, a))$, where $A_{m+1}=A_{1}, \omega \in \Omega$ and $\theta \in \Theta$.

Theorem 3.3 Let $(X, d)$ be a $b_{2}$-metric space, $A_{1}, A_{2}, \cdots, A_{m}$ are all nonempty subsets of $X=\bigcup_{i=1}^{m} A_{i}$. Let $f: X \rightarrow X$ be cyclic weaker $(\omega, \theta)$ -contraction in $b_{2}$-metric space, then $f$ has a unique fixed point in $\bigcap_{i=1}^{M} A_{i}$.

Proof Let $x_{0}$ be an arbitrary point in $X$ and we define a sequence $\left\{x_{n}\right\}$ by $x_{n+1}=f x_{n}=f^{n+1} x_{0}$, for all $n \in N$, if there exists some $n_{0} \in N$ such that $f x_{n_{0}-1}=f x_{n_{0}}$ then $f x_{n_{0}}=f x_{n_{0}}$. Thus $x_{n_{0}}$ is a fixed point of $f$. Suppose that $f x_{n-1} \neq f x_{n}$ for all $n \in N$, we know that there exists $i_{n} \in 1,2, \cdots, m$ such that $x_{n-1} \in A_{i_{n}}$ and $x_{n} \in A_{i_{n+1}}$ for any $n>0$. Since $f: X \rightarrow X$ be cyclic weaker $(\omega, \theta)$-contraction, we get

$$
\begin{aligned}
d\left(x_{n}, x_{n+1}, a\right) & =d\left(f x_{n-1}, f x_{n}, a\right) \leq \omega\left(d\left(x_{n-1}, x_{n}, a\right)\right)-\theta\left(d\left(x_{n-1}, x_{n}, a\right)\right) \\
& \leq \omega\left(d\left(x_{n-1}, x_{n}, a\right)\right) \\
& \leq \omega\left(\omega\left(d\left(x_{n-1}, x_{n-2}, a\right)\right)\right)=\omega^{2}\left(d\left(x_{n-1}, x_{n-2}, a\right)\right) \\
& \leq \cdots \\
& \leq \omega^{n}\left(d\left(x_{0}, x_{1}, a\right)\right) .
\end{aligned}
$$

Since sequence $\left\{\omega^{n}\left(d\left(x_{0}, x_{1}, a\right)\right)\right\}$ is decreasing for all $n \in N$, and this sequence must converge to some $\rho \geq 0$. We get $\rho=0$ by the following assumption.

First we assume that $\rho>0$, since $\omega$ is defined as a weaker Meir-Keeler function, there exists $\delta$ such that $\rho \leq d\left(x_{0}, x_{1}, a\right)<\delta+\rho$ for $x_{0}, x_{1} \in X$, there exists $n_{0} \in N$ such that $\omega^{n}\left(d\left(x_{0}, x_{1}, a\right)\right)<\rho$, from

$\lim _{n \rightarrow \infty} \omega^{n} d\left(x_{0}, x_{1}, a\right)=\rho$, we know that there exists $p_{0} \in N$ such that $\stackrel{n \rightarrow \infty}{\rho<\omega^{p}}\left(d\left(x_{0}, x_{1}, a\right)\right)<\rho+\delta$, for all $p \geq p_{0}$. Thus we get a conclusion $\omega^{p_{0}+n_{0}}\left(d\left(x_{0}, x_{1}, a\right)\right)<\rho$, which is a contraction. Thus $\lim _{n \rightarrow \infty} \omega^{n} d\left(x_{0}, x_{1}, a\right)=0$, and that is, $\lim _{n \rightarrow \infty} d\left(x_{n}, x_{n+1}, a\right)=0$.

Now we prove that $\left\{x_{n}\right\}$ is a Cauchy sequence. 
Suppose to the contrary, that is, $\left\{x_{n}\right\}$ is not a Cauchy sequence. Then there exists $\varepsilon>0$ for which we can find two sub sequences $\left\{n_{i}\right\}$ and $\left\{m_{i}\right\}$ such that $i<m_{i}<n_{i}$ and

$$
d\left(x_{m_{i}}, x_{n_{i}}, a\right) \geq \varepsilon \text { and } d\left(x_{m_{i}}, x_{n_{i}-1}, a\right)<\frac{\varepsilon}{S}<\varepsilon
$$

From the part 4 of Definition 3.6 and (3.6), we get

$$
\begin{aligned}
d\left(x_{m_{i}}, x_{n_{i}}, a\right) & \leq s\left[d\left(x_{m_{i}}, x_{m_{i}+1}, a\right)+d\left(x_{m_{i}+1}, x_{n_{i}}, a\right)+d\left(x_{m_{i}}, x_{n_{i}}, x_{m_{i}+1}\right)\right] \\
& \leq s\left[d\left(x_{m_{i}}, x_{m_{i}+1}, a\right)+d\left(x_{m_{i}+1}, x_{n_{i}}, a\right)\right]
\end{aligned}
$$

Taking $i \rightarrow \infty$, from (3.6) and (3.12) we have

$$
\frac{\varepsilon}{s} \leq \lim _{n \rightarrow \infty} d\left(x_{m_{i}+1}, x_{n_{i}}, a\right)
$$

Now by using the condition that $f$ is a cyclic weaker $(\omega, \theta)$-contraction, we get

$$
\begin{aligned}
d\left(x_{m_{i}+1}, x_{n_{i}}, a\right) & =d\left(x_{m_{i}}, x_{n_{i}-1}, a\right) \\
& \leq \omega\left(d\left(x_{m_{i}}, x_{n_{i}-1}, a\right)\right)-\theta\left(d\left(x_{m_{i}}, x_{n_{i}-1}, a\right)\right) \\
& \leq \omega\left(d\left(x_{m_{i}}, x_{n_{i}-1}, a\right)\right)
\end{aligned}
$$

Letting $i \rightarrow \infty$ and using the condition of $\omega$, we get

$$
\lim _{n \rightarrow \infty} d\left(x_{m_{i}+1}, x_{n_{i}}, a\right)<\frac{\varepsilon}{s}
$$

From (3.13) and (3.14) $\frac{\varepsilon}{s} \leq \lim _{n \rightarrow \infty} d\left(x_{m_{i}+1}, x_{n_{i}}, a\right)<\frac{\varepsilon}{s}$, which is a contraction. Therefore $\left\{x_{n}\right\}$ is a Cauchy sequence in $X$.

Since $X$ is a complete set, there exists a point $z \in \bigcup_{i=1}^{m} A_{i}$ such that $n \rightarrow \infty$, $\left\{x_{n}\right\} \rightarrow z$. For $X=\bigcup_{i=1}^{m} A_{i}$ is a cyclic representation of $X$ respect to $f$, thus in each $A_{i}$ for $i \in\{1,2, \cdots, m\}$, the sequence $\left\{x_{n}\right\}$ has infinite term. A sub sequence $\left\{x_{n_{k}}\right\}$ of $\left\{x_{n}\right\}$, we take this sub sequence and it also all converge to $Z$, for all $i=1,2, \cdots, m$. Since

$$
\begin{aligned}
d\left(x_{n_{k}+1}, f z, a\right) & =d\left(f x_{n_{k}}, f z, a\right) \\
& \leq \omega\left(d\left(x_{n_{k}}, z, a\right)\right)-\theta\left(d\left(x_{n_{k}}, z, a\right)\right) \\
& \leq \omega\left(d\left(x_{n_{k}}, z, a\right)\right),
\end{aligned}
$$

From the above inequality, letting $k \rightarrow \infty$, we get $d(z, f z, a)=0$, so $z=f z$.

Now we prove the fixed point is unique for $f$. Suppose there exists another fixed point $y$, since $f$ gets the cyclic character, we have $z, y \in \bigcup_{i=1}^{m} A_{i}$. Since $f$ is a cyclic weaker $(\omega, \theta)$-contraction, we get

$$
\begin{aligned}
d(z, y, a) & =d(z, f y, a) \\
& =\lim _{n \rightarrow \infty} d\left(x_{n_{k}+1}, f y, a\right)=\lim _{n \rightarrow \infty} d\left(f x_{n_{k}}, f y, a\right) \\
& \leq \lim _{n \rightarrow \infty}\left[\omega\left(d\left(x_{n_{k}}, y, a\right)\right)-\theta\left(d\left(x_{n_{k}}, y, a\right)\right)\right] \\
& \leq d(z, a, y)-\theta(d(y, z, a)),
\end{aligned}
$$


then we get

$\theta(d(y, z, a))=0$, that is $y=z$, we get the result of the uniqueness of point

\section{Conflicts of Interest}

The authors declare no conflicts of interest regarding the publication of this paper.

\section{References}

[1] Banach, S. (1992) Sur les opérations dans les ensembles abtraits et leur applications aux équations intégrales. Fundamenta Mathematicae, 3, 133-181. https://doi.org/10.4064/fm-3-1-133-181

[2] Meri, A. and Keeler, E. (1969) A Theorem on Contraction Mappings. Journal of Mathematical Analysis and Applications, 28, 326-329.

https://doi.org/10.1016/0022-247X(69)90031-6

[3] Chistyakov, W. (2010) Modular Metric Space, I: Basic Concepts. Nonlinear Analysis, 72, 1-14. https://doi.org/10.1016/j.na.2009.04.057

[4] Piao, Y.J. (2008) Unique Common Fixed Point for a Family of Self-Maps with Same Type Contractive Condition in 2-Metric Spaces. Analysis in Theory and Applications, 24, 316-320. https://doi.org/10.1007/s10496-008-0316-9

[5] Piao, Y.J. (2012) Unique Common Fixed Point Fir a Family of Mappings with $\phi$ -Contractive Type in 2-Metric Spaces. Applied Mathematics, 3, 73-77. https://doi.org/10.4236/am.2012.31012

[6] Piao, Y.J. and Jin, Y.F. (2012) New Unique Common Fixed Results for Four Mappings with $\phi$-Contractive Type in 2-Metric Spaces. Applied Mathematics, 3, 734-737. https://doi.org/10.4236/am.2012.37108

[7] Czerwik, S. (1993) Contraction Mappings in b-Metric Spaces. Acta Mathematica et Informatica Universitatis Ostraviensis, 1, 5-11.

[8] Czerwik, S. (1998) Nonlinear Set-Valued Contraction Mappings in b-Metric Spaces. Atti del Seminario Matematico e Fisico dell' Universita di Modena, 46, 263-276.

[9] Mustafa, Z., Parvaech, V., Roshan, J.R. and Kadelburg, A. (2014) $b_{2}$-Metric Spaces and Some Fixed Point Theorems. Fixed Point Theory and Applications, 144.

https://doi.org/10.1186/1687-1812-2014-144 